\title{
Journal of ELECTROSTATICS
}

\section{Dielectrophoretic manipulation of rod-shaped viral particles}

\author{
Hywel Morgan*, Nicolas G. Green \\ Bioelectronics Research Centre, University of Glasgow, Glasgow, UK GI2 $8 Q Q$
}

Received 19 February 1997; received in revised form 17 June 1997; accepted 17 June 1997

\begin{abstract}
The dielectrophoretic manipulation of a rod-shaped virus, tobacco mosaic virus (TMV) by means of non-uniform AC electric fields is described. An expression is derived for the dielectrophoretic force on a homogeneous dielectric cylinder suspended in a liquid medium which shows that the dielectrophoretic force varies over a wide range of frequencies and medium conductivities. Measurements of the dielectrophoretic behaviour of TMV particles in microelectrode arrays have been made as a function of frequency and applied field strength. The results are shown to be in quantitative agreement with the derived expression for the dielectrophoretic force. By measuring the threshold field strength for the onset of dielectrophoresis, the force on a single TMV particle has been estimated. The results point to the potential use of dielectrophoresis for the collection and manipulation of sub-micron particles using microelectrode arrays. (C) 1997 Elsevier Science B.V.
\end{abstract}

Keywords: Dielectrophoresis; Viral particles; Tobacco mosaic virus; Micro-electrodes; Submicron particle collection

\section{Introduction}

The manipulation and identification of sub-cellular components is becoming increasingly important in modern biotechnology. For example, current diagnostic techniques rely on analysing material from many millions of cells, but modern gene probing can, in principle, be performed on the DNA from a single cell. Also, the precise manipulation of genetic material into and out of single cells is not possible using conventional techniques. Therefore, new methods for the non-destructive characterisation, separation and fractionation of sub-cellular bioparticles, such as chromosomes, plasmids, viruses and macromolecules must be developed.

The manipulation of cells and microorganisms using non-uniform $\mathrm{AC}$ electric fields has shown great promise in recent years. First investigated by Pohl as long ago as

*Corresponding author. Tel.: + 44141330 5237; fax: + 44141330 4907; e-mail: h.morgan@elec.gla.ac.uk. 
$1951[1,2]$, the technology has developed rapidly over the last decade, primarily due to the development of new methods for producing miniaturised electrodes [3]. Dielectrophoresis is defined as the motion of polarisable particles in non-uniform, alternating electric fields. In general, a biological particle will polarise under the influence of an electric field and experience a translational force. The force can direct particles either towards regions of high electric field strength (positive DEP) or low electric field strength (negative DEP) depending on the relative differences between the polarisability of the particle and the medium [4-6]. The magnitude of the dielectrophoretic force depends on the dielectric properties of the particle and as a consequence DEP has been used to identify and separate particles, such as cells, which are apparently identical except for subtle differences in age or composition [7].

The dielectrophoretic force scales with the volume of the particle and it had been assumed until recently that the movement of particles smaller than $1 \mu \mathrm{m}$ in diameter was impossible because of the effects of Brownian motion. For a moderate field strength (around $10^{4} \mathrm{~V} / \mathrm{m}$ ) it has been shown [8] that, as the particle size is reduced, the effect of thermal forces increases correspondingly. This implies that for particles of less than $1 \mu \mathrm{m}$ in diameter, the dielectrophoretic force would be too low to induce deterministic movement. However, this simple argument does not take into account the extremely high electric fields that can be generated using micro- and nanofabricated electrode structures. Using such electrode structures, the dielectrophoretic trapping and manipulation of viruses, macromolecules and sub-micron latex particles has now been unequivocally demonstrated [9-14]. In this paper we show that it is possible to dielectrophoretically manipulate a rod-shaped virus, specifically tobacco mosaic virus (TMV) using nano-fabricated electrode arrays. Virions can be induced to move towards or away from regions of high electric field strength demonstrating both positive and negative DEP. An expression for the dielectrophoretic force on a dielectric cylinder is derived and it is shown that experimental results for the frequencydependent behaviour and magnitude of the DEP force are in qualitative agreement with this theory.

\section{Theory}

\subsection{Calculation of dielectrophoretic force}

The dielectrophoretic force $\bar{F}(t)$ on a particle is given by the following general expression:

$$
\bar{F}(t)=(\bar{m}(t) \cdot \nabla) \bar{E}(t)
$$

where $\bar{m}(t)$ is the induced dipole moment of the particle, $\bar{E}(t)$ the electric field and $\nabla$ is the del vector operator. For a particle of volume $v$, this equation can also be written in terms of the effective polarisability $u$ as

$$
\bar{F}(t)=v u(\bar{E}(t) \cdot \nabla) \bar{E}(t)=\frac{v u}{2} \nabla E(t)^{2}
$$


For a homogeneous uncharged sphere, the effective polarisability is given by the following well-known equation:

$$
u=\varepsilon_{\mathrm{m}}\left\{f\left(\varepsilon_{\mathrm{p}}^{*}, \varepsilon_{\mathrm{m}}^{*}\right)\right\}
$$

where

$$
f\left(\varepsilon_{\mathrm{p}}^{*}, \varepsilon_{\mathrm{m}}^{*}\right)=\left(\frac{\varepsilon_{\mathrm{p}}^{*}-\varepsilon_{\mathrm{m}}^{*}}{\varepsilon_{\mathrm{p}}^{*}+2 \varepsilon_{\mathrm{m}}^{*}}\right)
$$

the Clausius Mossotti [15-17] factor, $\varepsilon_{\mathrm{m}}^{*}$ and $\varepsilon_{\mathrm{p}}^{*}$ are the complex permittivities of the medium and the particle of radius $a$, respectively. A general complex permittivity is given by $\varepsilon^{*}=\varepsilon-\mathrm{j}(\sigma / \omega)$ where $\varepsilon$ is the real permittivity, $\sigma$ the conductivity, $\mathrm{j}=\sqrt{-1}$ and $\omega$ the angular frequency.

Combining Eqs. (2) and (3) gives the well-known expression for the dielectrophoretic force on a sphere:

$$
\bar{F}(t)=2 \pi a^{3} \varepsilon_{\mathrm{m}} \operatorname{Re}\left\{\left(\frac{\varepsilon_{\mathrm{p}}^{*}-\varepsilon_{\mathrm{m}}^{*}}{\varepsilon_{\mathrm{p}}^{*}+2 \varepsilon_{\mathrm{m}}^{*}}\right) \nabla E(t)^{2}\right\},
$$

where $\operatorname{Re}\{\}$ indicates the real part of the expression in brackets. For an ellipsoid, the dipole moment is also a function of the axes of the ellipsoid. For an ellipsoid as shown in Fig. 1, the induced dipole moment $m_{x}(t)$ and complex polarisability $u_{x}$ of a solid homogeneous dielectric ellipsoid, where $x$ is one of the coordinate axes, has been derived $[18,19]$ :

$$
\begin{aligned}
& m_{x}(t)=(4 \pi a b c / 3) \varepsilon_{\mathrm{m}} \chi_{\alpha}(t) E_{0}, \quad \alpha=x, y, z, \\
& u_{\alpha}=\varepsilon_{\mathrm{m}} \chi_{\alpha}(t), \quad \alpha=x, y, z,
\end{aligned}
$$

where

$$
\chi_{\alpha}(t)=\frac{\varepsilon_{\mathrm{p}}^{*}-\varepsilon_{\mathrm{m}}^{*}}{\left(\varepsilon_{\mathrm{p}}^{*}-\varepsilon_{\mathrm{m}}^{*}\right) A_{\alpha}+\varepsilon_{\mathrm{m}}^{*}}, \quad \alpha=x, y, z
$$

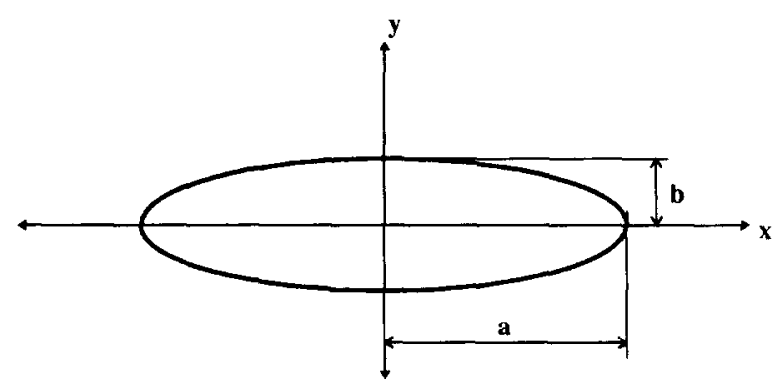

Fig. 1. Diagram of a prolate ellipsoid with complex permittivity, $\varepsilon_{\mathrm{p}}^{*}$ suspended in a medium of complex permittivity $\varepsilon_{\mathrm{m}}^{*}$. 
and $\varepsilon_{\mathrm{p}}^{*}$ is the complex dielectric constant of the solid body and $\varepsilon_{\mathrm{m}}^{*}$ is the complex dielectric constant of the suspending medium. $A_{\alpha}$ is the depolarising factor along $\alpha$ and is given by

$$
A_{\alpha}=\frac{1}{2} a b c \int_{0}^{\infty} \frac{\mathrm{d} s}{\left(s+i_{\alpha}^{2}\right) R}, \quad i_{x}=a, i_{y}=b, i_{z}=c,
$$

where

$$
R=\sqrt{\left(s+a^{2}\right)+\left(s+b^{2}\right)+\left(s+c^{2}\right)} .
$$

Taking a prolate spheroid with $a>b=c$, and assuming that the applied field, $\bar{E}_{0}$ is parallel to the major axis $a$ and that both are orientated along the $x$-axis, then the integral of Eq. (8) is given by the following expression [18]:

$$
\int_{0}^{\infty} \frac{\mathrm{d} s}{\left(s+b^{2}\right)\left(s+a^{2}\right)^{3 / 2}}=-\frac{1}{a^{3} e^{3}}\left(2 e-\ln \frac{1+e}{1-e}\right),
$$

where the eccentricity is $e=\sqrt{1-\left(b^{2} / a^{2}\right)}$. For $a \gg b$ then $e \rightarrow 1$, i.e. as the spheroid degenerates into a long, needle-shaped cavity, the depolarising factor $A_{x}$ approaches zero. Thus, at any point inside the cavity the internal field $\bar{E}_{\mathrm{in}}=\bar{E}_{0}$.

For the case of a rod aligned with the field, putting $A_{x} \rightarrow 0$ in Eqs. (5) and (6) gives

$$
u(t)=\varepsilon_{\mathrm{m}} \frac{\varepsilon_{\mathrm{p}}^{*}-\varepsilon_{\mathrm{m}}^{*}}{\varepsilon_{\mathrm{m}}^{*}} .
$$

The volume of an ellipsoid is $v=\frac{4}{3} \pi a b c$ and substituting this and Eq. (11) into Eq. (2) gives the dielectrophoretic force on a rod whose major axis is parallel to the electric field as

$$
\bar{F}(t)=\frac{2 \pi a b c}{3} \varepsilon_{\mathrm{m}} \operatorname{Re}\left\{\frac{\varepsilon_{\mathrm{p}}^{*}-\varepsilon_{\mathrm{m}}^{*}}{\varepsilon_{\mathrm{m}}^{*}}\right\} \nabla \bar{E}^{2} .
$$

The derivation of the force on multi-shelled particles simply involves replacing $\varepsilon_{\mathbf{p}}^{*}$ with the relevant complex permittivity for each shell [19].

An ellipsoid initially at random orientation to a static electric field will experience a torque tending to align one of the three axes with the field. The ellipsoid will align so that all components of torque are zero, constituting three directions of equilibrium [18]. The stability of the ellipsoid in any one of three orientations depends on the relative magnitudes of the depolarising factors and on the relative magnitudes of $a, b$, and $c$, the ellipsoid axes. Miller and Jones [20] have shown that the orientation of ellipsoidal cells can be controlled by the conductivity of the medium and the frequency of the applied field. The ellipse can orient with the major axis parallel or perpendicular to the applied field and given values of the depolarisation factors, $A_{\alpha}$ for each axis, it was shown that experimental observations were in accord with theory. The sign of the torque on the three axes of TMV was calculated as outlined by Miller and Jones [20]. The virus was modelled as a prolate ellipsoid with the ratios of major to minor axes, $a / b=15.5$, and the depolarisation factors calculated from Eq. (10) giving $A_{x}=0.0102$ 
and $A_{y}=A_{z}=\frac{1}{2}\left(1-A_{x}\right)=0.4949$. It was found that, for conductivities greater than $0.1 \mathrm{Sm}^{-1}$ and for all frequencies, the sign of the torque was always to orient the major axis ( $x$-axis) parallel to the applied field. All other orientations ( $y$ and $z$ parallel to the field) were unstable positions with the absolute value of torque $=0$. Small perturbations around these points caused realignment of the particle with the long axis parallel to the field. For the particular case of the TMV it was also found that reorientation of the particle could occur in a narrow frequency window $(5-50 \mathrm{MHz})$ for conductivities less than $0.1 \mathrm{~S} \mathrm{~m}^{-1}$. Thus, for our range of experimental conditions we can assume that the major axis will always align with the field, so that Eq. (12) will be valid for the general case of the dielectrophoretic behaviour of a long rod.

\subsection{Calculations}

The relative dielectrophoretic force on a long cylindrical rod with the dimensions of a tobacco mosaic virus was calculated as a function of applied frequency and medium conductivity. TMV is a cylindrical unenveloped virus, $280 \mathrm{~nm}$ long and $18 \mathrm{~nm}$ in diameter consisting of a protein shell surrounding a core of RNA [21]. To model its dielectric behaviour it was considered to be a homogeneous dielectric rod with single value of permittivity and conductivity. For the simulations a value of 60 was assigned to the particle's permittivity and $0.1 \mathrm{~S} \mathrm{~m}^{-1}$ to the conductivity. This high value of particle conductivity is due to the combined effects of bulk conductivity and surface conductance effects. Both these permittivity and conductivity values are in approximate agreement with our own dielectric measurements on TMV (unpublished data) and with dielectric measurements of alfalfa virus [22].

The results of the simulations are shown in Figs. 2(a) and (b). These plots show how the factor $\operatorname{Re}\left\{\left(\varepsilon_{\mathrm{p}}^{*}-\varepsilon_{\mathrm{m}}^{*}\right) / \varepsilon_{\mathrm{m}}^{*}\right\}$ varies with conductivity of the suspending medium and frequency of applied field. Fig. 2(b) is an enlargement of the central section of Fig. 2(a) and illustrates how the force rapidly diminishes with increasing frequency. At high frequencies $\omega \varepsilon / \sigma \gg 1$ and

$$
\left(\frac{\varepsilon_{\mathrm{p}}^{*}-\varepsilon_{\mathrm{m}}^{*}}{\varepsilon_{\mathrm{m}}^{*}}\right) \rightarrow\left(\frac{\varepsilon_{\mathrm{p}}-\varepsilon_{\mathrm{m}}}{\varepsilon_{\mathrm{m}}^{*}}\right) \approx-1 \text { for } \varepsilon_{\mathrm{p}}<\varepsilon_{\mathrm{m}} .
$$

Conversely, for low frequencies $\omega \varepsilon / \sigma \ll 1$ and $\left\{\left(\varepsilon_{\mathrm{p}}^{*}-\varepsilon_{\mathrm{m}}^{*}\right) / \varepsilon_{\mathrm{m}}^{*}\right\} \rightarrow\left\{\left(\sigma_{\mathrm{p}}-\sigma_{\mathrm{m}}\right) / \sigma_{\mathrm{m}}\right\}$ which, approximates to $\approx \sigma_{\mathrm{p}} / \sigma_{\mathrm{m}}$ for low medium conductivities. For our calculations assuming $A_{x} \rightarrow 0$, then at low frequencies and in a low conductivity medium $\sigma_{\mathrm{p}} / \sigma_{\mathrm{m}}$ may reach $10^{4}$ within our experimental conditions. In practice, $a / b=15.5$ for TMV, giving $A_{x}=0.0102$ so that

$$
\chi_{x}(t)=\frac{\varepsilon_{\mathrm{p}}^{*}-\varepsilon_{\mathrm{m}}^{*}}{\left(\varepsilon_{\mathrm{p}}^{*}-\varepsilon_{\mathrm{m}}^{*}\right) 0.0102+\varepsilon_{\mathrm{m}}^{*}}
$$

and in the low frequency and conductivity limit $\chi_{x}(t) \rightarrow 1 / 0.0102 \approx 100$. By contrast the polarisation factor for a sphere (Clausius Mossotti factor) is bounded by the limits $1>f\left(\varepsilon_{\mathrm{p}}^{*}, \varepsilon_{\mathrm{m}}^{*}\right)>-\frac{1}{2}$ indicating that the force on a rod may be many times greater than that on a sphere of equivalent dimensions. 

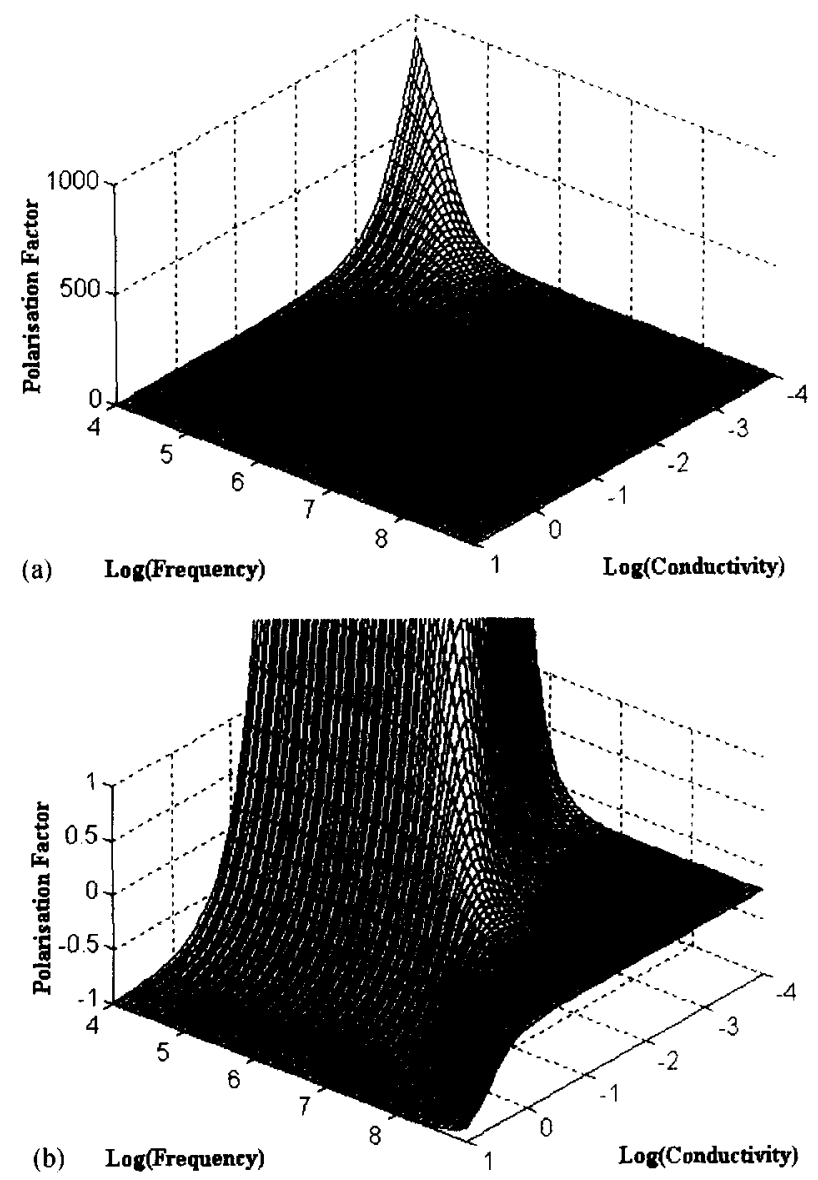

Fig. 2. Three-dimensional plot showing how the factor $\operatorname{Re}\left\{\left(\varepsilon_{\mathrm{p}}^{*}-\varepsilon_{\mathrm{m}}^{*}\right) / \varepsilon_{\mathrm{m}}^{*}\right\}$ (polarisation factor, with depolarisation factor $A_{x} \rightarrow 0$ ) varies with the conductivity of the suspending medium and frequency of applied field for a particle with $\varepsilon_{\mathrm{p}}=60, \sigma_{\mathrm{p}}=0.1 \mathrm{~S} \mathrm{~m}^{-1}$. (b) Enlargement of the central section of (a).

Fig. 3 shows how the relative polarisation factor $\operatorname{Re}\left\{\left(\varepsilon_{\mathrm{p}}^{*}-\varepsilon_{\mathrm{m}}^{*}\right) / \varepsilon_{\mathrm{m}}^{*}\right\}$ varies with the conductivity of the medium over the range $10^{-3}-10^{1} \mathrm{~S} \mathrm{~m}^{-1}$, at a constant frequency of $100 \mathrm{kHz}$. It can be seen that the force on the particle is close to zero at high conductivities but increases rapidly for conductivities below $10^{-2} \mathrm{~S} \mathrm{~m}^{-1}$. The crossover frequency from positive DEP to negative DEP is the frequency at which $\operatorname{Re}\left\{\left(\varepsilon_{\mathrm{p}}^{*}-\varepsilon_{\mathbf{m}}^{*}\right) / \varepsilon_{\mathrm{m}}^{*}\right\}=0$ and the net force is zero. Fig. 4 shows how this frequency varies with medium conductivity over the range from $10^{-3}$ to $1 \mathrm{~S} \mathrm{~m}^{-1}$ for a rod-shaped particle with $\varepsilon_{\mathrm{p}}=60, \sigma_{\mathrm{p}}=0.1 \mathrm{~S} \mathrm{~m}^{-1}$. The trend shows a linear increase in crossover frequency then an abrupt change at just below $10^{-1} \mathrm{~S} \mathrm{~m}^{-1}$, where at this conductivity the dielectrophoretic response of the virus is wholly negative. 


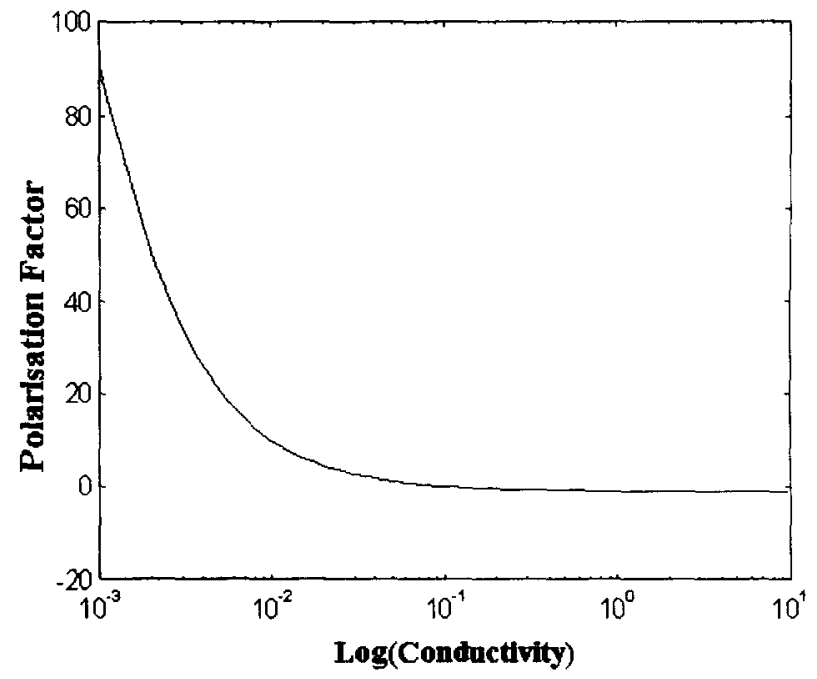

Fig. 3. A plot of the polarisation factor $\operatorname{Re}\left\{\left(\varepsilon_{\mathrm{p}}^{*}-\varepsilon_{\mathrm{m}}^{*}\right) / \varepsilon_{\mathrm{m}}^{*}\right\}$ as a function of medium conductivity at a constant frequency of $100 \mathrm{kHz}$. The plot was calculated with the following parameters for the particle $\varepsilon_{\mathrm{p}}=60, \sigma_{\mathbf{p}}=0.1 \mathrm{~S} \mathrm{~m}^{-1}$.

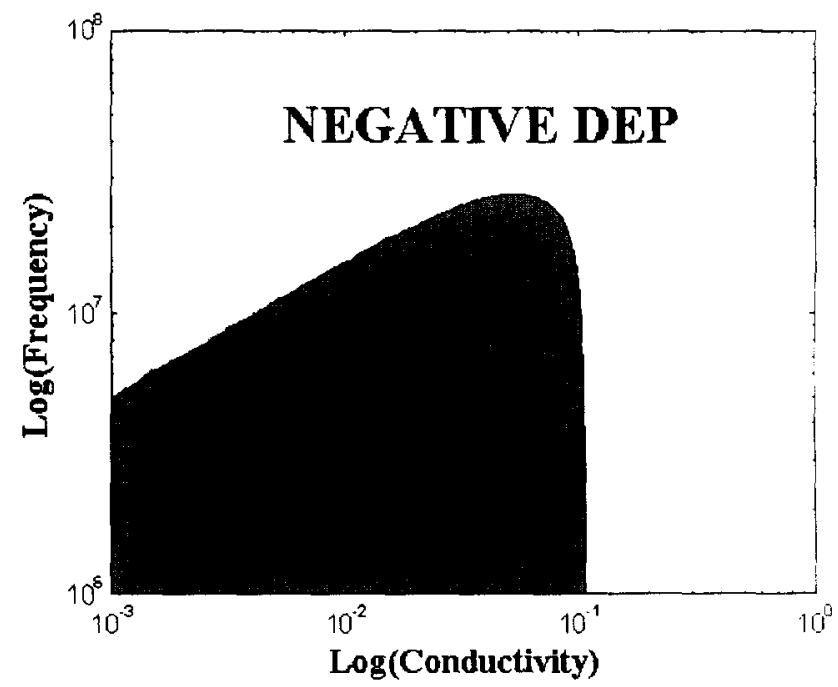

Fig. 4. Variation in the crossover frequency (frequency at which $\operatorname{Re}\left\{\left(\varepsilon_{\mathrm{p}}^{*}-\varepsilon_{\mathrm{m}}^{*}\right) / \varepsilon_{\mathrm{m}}^{*}\right\}=0$ and the net force is zero) as a function of medium conductivity over the range $10^{-3}$ to $1 \mathrm{~S} \mathrm{~m}^{-1}$. The plot asymptotes to a value of conductivity of just below $10^{-1} \mathrm{~S} \mathrm{~m}^{-1}$. For higher conductivities the DEP response of the virus is completely negative for all frequencies. 


\section{Experimental}

\subsection{Electrode fabrication}

Electrodes were manufactured on glass microscope slides using standard photoand electron-beam lithographic techniques [3]. Electrodes, consisting of layers of $10 \mathrm{~nm}$ titanium, $10 \mathrm{~nm}$ palladium and $100 \mathrm{~nm}$ gold, with minimum feature sizes in the range $0.5-5 \mu \mathrm{m}$ were fabricated. Sawtooth-shaped electrodes were used for trapping of TMV and Fig. 5 shows a scanning electron microscope photograph of a typical electrode array. This electrode was manufactured with $4 \mu \mathrm{m}$ gaps using direct-write electron beam lithography to give a well-defined and consequently precise field

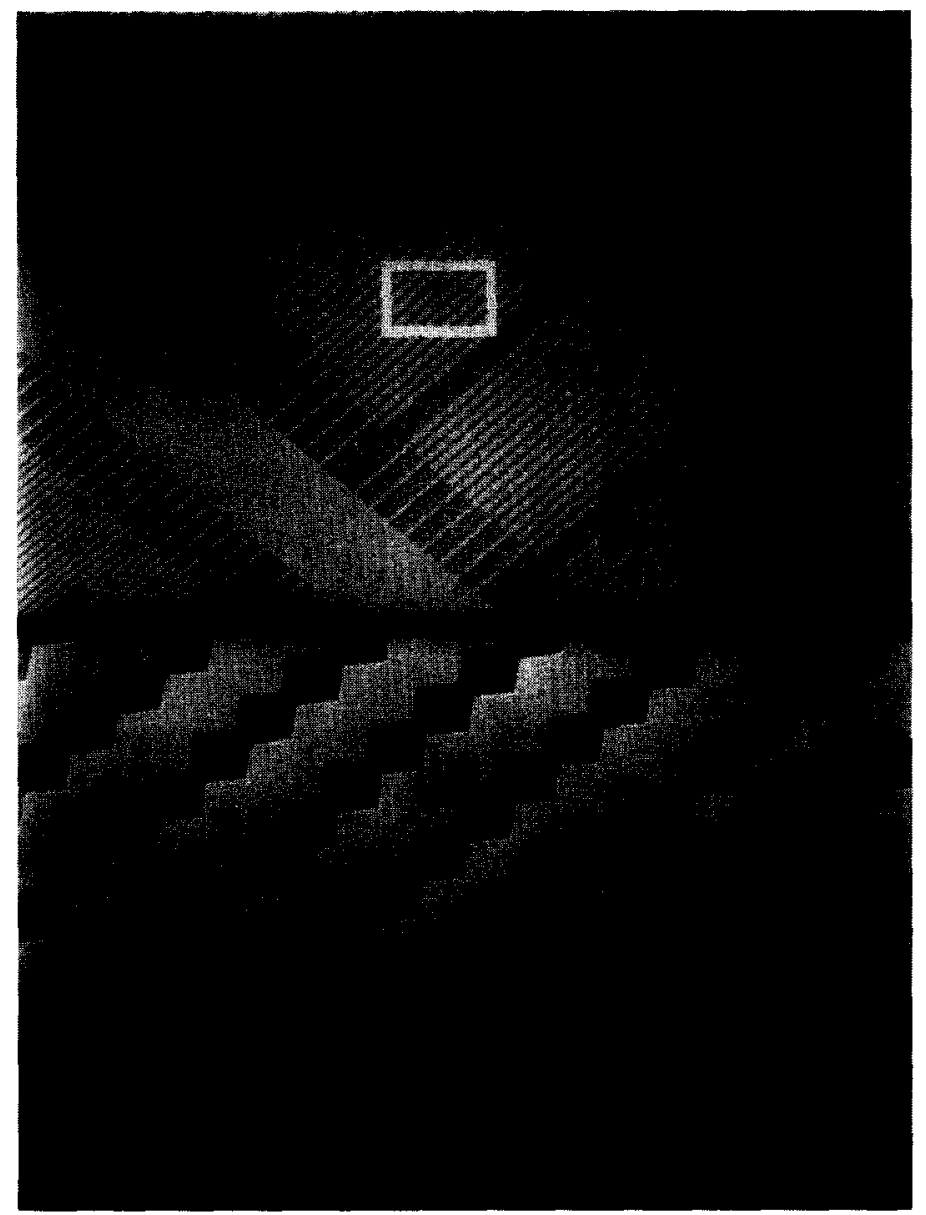

Fig. 5. A scanning electron microscope image of the sawtooth electrode array used for dielectrophoretic trapping of TMV. A typical electrode array covers an area of several $\mathrm{mm}^{2}$. 
geometry and high field gradients. Typical electrode arrays covered an area of $10 \times 10 \mathrm{~mm}$ and were located in the central part of a microscope slide. The virus suspension $(10 \mu \mathrm{l})$ was pipetted onto the array and covered with a glass cover slip. Movement of particles was observed using a fluorescence microscope (Nikon Microphot). Images were recorded using a video camera and S-VHS recorder, before digitising with a PC. The electric field was generated using a Direct Digital Synthesis frequency generator under computer control giving $10 \mathrm{~V}$ peak-to-peak at frequencies up to $20 \mathrm{MHz}$.

\subsection{Preparation of virus}

The preparation and fluorescent labelling of the virus has been described in detail elsewhere [23]. Labelled virus was concentrated by ultracentrifugation for $1 \mathrm{~h}$ at $200000 \mathrm{~g}$ and re-suspended in potassium phosphate buffer, pH 7.2. For the DEP experiments, a range of solution conductivities were used, obtained by varying the concentration of potassium phosphate in the buffer over the range $0.1-100 \mathrm{mM}$.

\section{Results}

Trapping of TMV was achieved using the sawtooth electrode array of Fig. 5. This type of array generates an extremely high electric field gradient at the tips of the teeth approaching $10^{21} \mathrm{~V}^{2} / \mathrm{m}^{3}$. A black and white photograph of the array after trapping TMV particles is shown in Fig. 6. The frequency used to obtain this image was $100 \mathrm{kHz}$ and the applied voltage was $4 \mathrm{~V}$ peak to peak (corresponding to an average field strength of $1 \times 10^{6} \mathrm{~V} / \mathrm{m}$ ). It was not possible to observe single virions, only a faint haze which continually increased in brightness as more virions become trapped. Occasional large clumps of virus were observed at the electrodes. Positive DEP of TMV was observed over the frequency range $1 \mathrm{kHz}$ to $1.5 \mathrm{MHz}$ for medium conductivities in the range $170 \mu \mathrm{S} / \mathrm{cm}$ to $14 \mathrm{mS} / \mathrm{cm}$. Above $1.5 \mathrm{MHz}$ the virus did not collect at the electrode tips. At frequencies above approximately $20 \mathrm{MHz}$ and over all conductivities examined the TMV repelled from the electrodes under the action of negative DEP. The observation of the negative DEP was made easier by using positive DEP to collect particles at the electrode edges before switching frequency to the negative DEP region.

The crossover frequency was measured and this data plotted against medium conductivity. The results are shown in Fig. 7 (experimental points). Also shown in this figure is the best fit to these experimental points, obtained by modelling the particle as a rod with a relative permittivity, $\varepsilon_{\mathrm{p}}=55 \pm 2$ and conductivity, $\sigma_{\mathrm{p}}=0.085 \pm$ $0.002 \mathrm{~S} \mathrm{~m}^{-1}$, values which are close to the parameters used in the simulations.

Figs. 2 and 3 predict that at a frequency of $100 \mathrm{kHz}$, the force on the virions should vary with conductivity by approximately $10^{2}$. With our current experimental system it is difficult to measure the absolute force on the virion which would allow us to test this prediction. Therefore, the threshold field strength $E_{t}^{2}$ required to initiate observable DEP was measured as a function of medium conductivity. Movement of a single viral 


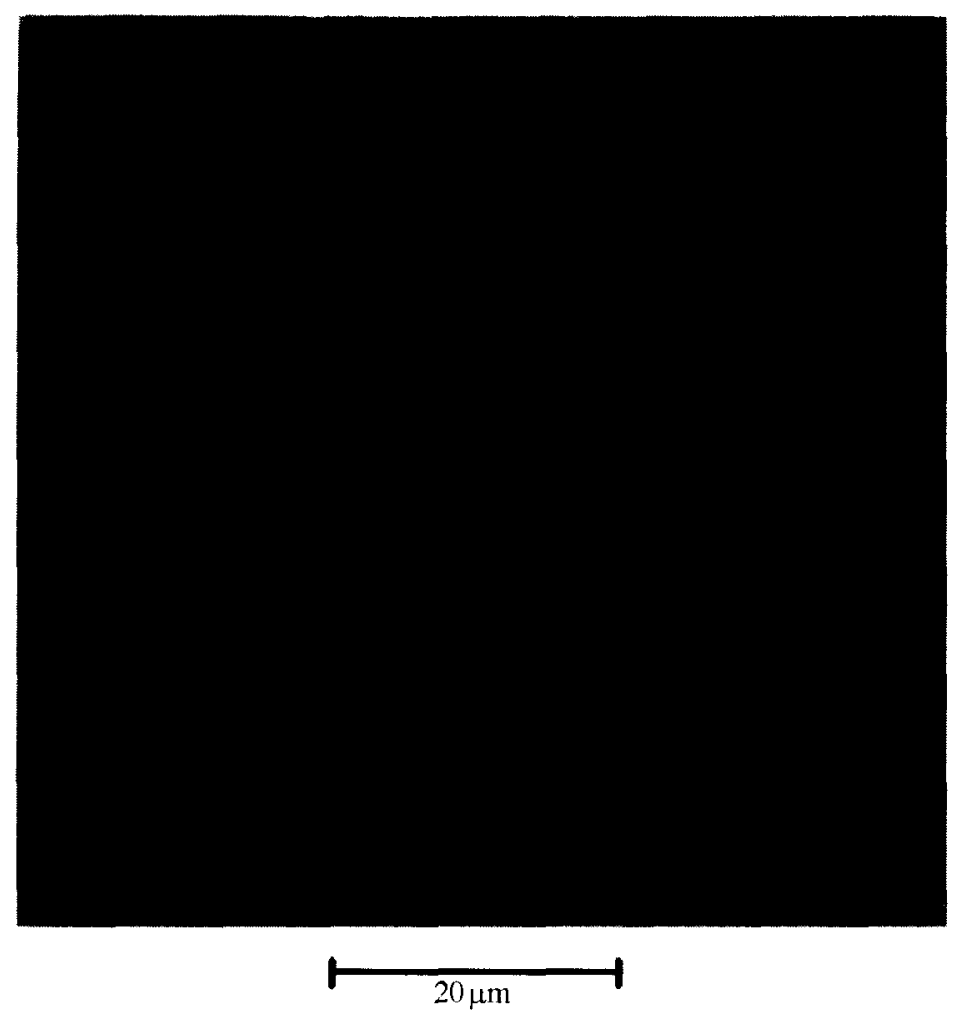

Fig. 6. A black and white fluorescence photograph of the sawtooth electrode array 1 min after application a voltage of $4 \mathrm{~V}$ peak to peak at $100 \mathrm{kHz}$. The trapping of TMV particles corresponds to the white haze at the tips of electrodes as shown in the picture.

particle could not be observed, but the collection of viruses was so fast (a white haze of light surrounding the electrode tips appeared in less than $1 \mathrm{~s}$ ) that it was possible to obtain a qualitative value for the threshold field. Over a conductivity range of $2 \times 10^{-3}$ to $2 \times 10^{-1} \mathrm{~S} \mathrm{~m}^{-1}$ the threshold field strength measured at a frequency of $100 \mathrm{kHz}$ increased from $2.5 \times 10^{5}$ to $2 \times 10^{6} \mathrm{~V} \mathrm{~m}^{-1}$, as predicted from Eq. (12).

At a conductivity of $10^{-2} \mathrm{~S} \mathrm{~m}^{-1}$ and above, no DEP was observed (although convective effects were observed at these high conductivities), confirming the theoretical prediction that the polarisation factor $\operatorname{Re}\left\{\left(\varepsilon_{\mathrm{p}}^{*}-\varepsilon_{\mathrm{m}}^{*}\right) / \varepsilon_{\mathrm{m}}^{*}\right\}$ is too small for any noticeable DEP at higher conductivities for the indicated field strength.

\section{Discussion}

Experiments determining the orientation of TMV by low-frequency AC fields were performed as long ago as $1939[24,25]$. However, these results were governed by 


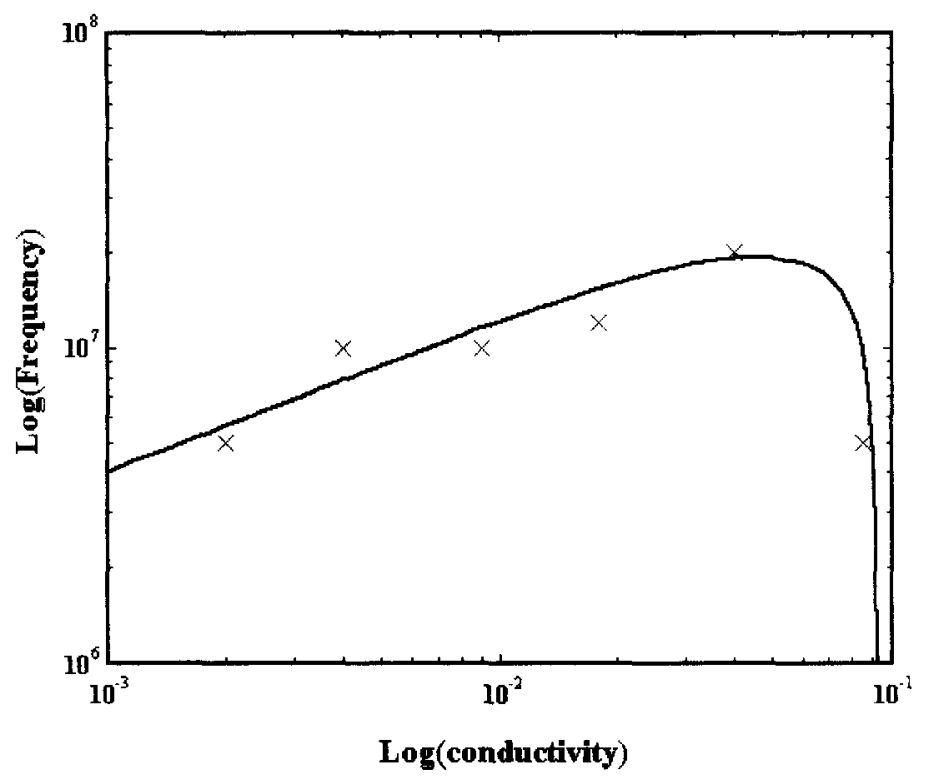

Fig. 7. Experimentally determined values of crossover frequency for the TMV plotted as a function of medium conductivity. Also shown in this figure is the best fit to the data, calculated by assigning a relative permittivity of $55 \pm 2$ and a conductivity of $0.085 \pm 0.002 \mathrm{~S} \mathrm{~m}^{-1}$ to the particle.

electrophoretic, rather than dielectrophoretic effects. Our work demonstrates that dielectrophoretic trapping of small particles can be easily achieved under the appropriate conditions, consistent with earlier observations of the trapping of enveloped viruses and latex beads [10-14]. However, the frequency-dependent nature of the force on a nano-scale particle has not been studied in detail previously. Our results clearly indicate that the dielectrophoretic properties of the virus are frequency dependent and, for the rod-shaped TMV, are governed by the variation in polarisation factor of Eq. (12). The observed transition from positive DEP to negative DEP (crossover frequency) plotted in Fig. 4 agrees remarkably well with the curve predicted from Eq. (12) with measured crossover frequencies spanning the range $5-20 \mathrm{MHz}$ and varying with medium conductivity. TMV is an unenveloped virus, therefore a singlevalue of conductivity and permittivity was assigned to the virion, as outlined in Section 2.2. The values of $\varepsilon_{\mathrm{p}}=55$ and $\sigma_{\mathrm{p}}=0.085 \mathrm{~S} \mathrm{~m}^{-1}$ produce excellent fits to the experimental data as shown in Fig. 7.

The absolute force on the TMV can be calculated from Eq. (12) given a knowledge of the electric field gradient, $\nabla E^{2}$. At the threshold field strength the DEP force becomes large enough to produce an observable motion of the virions. At a frequency of $100 \mathrm{kHz}$ and medium conductivity of $2 \times 10^{-3} \mathrm{~S} \mathrm{~m}^{-1}$ the threshold field strength, $E_{\mathrm{t}}=2.5 \times 10^{5} \mathrm{~V} \mathrm{~m}^{-1}$. The field gradient varies across the electrodes in a non-uniform manner and for the sawtooth electrodes the field gradient was calculated (using finite-element methods) as a function of distance between the tips for an applied 


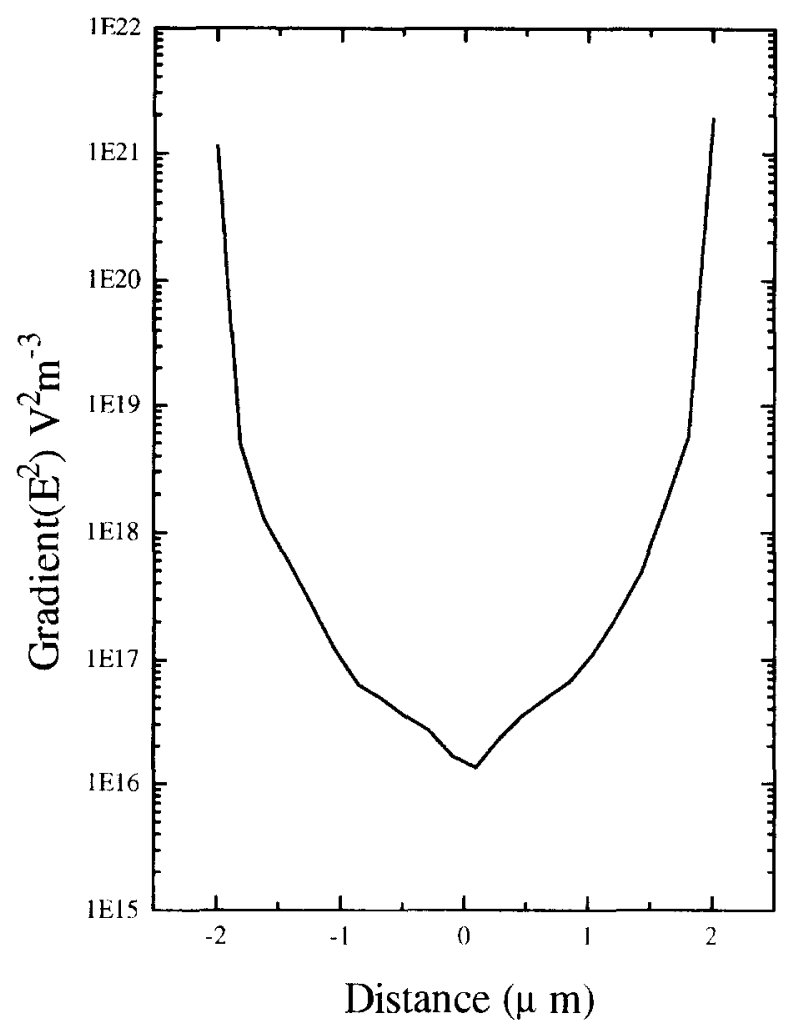

Fig. 8. The field gradient plotted as a function of distance between the tips for the sawtooth electrode. The applied voltage was $2 \mathrm{~V}$ and the tip separation $4 \mu \mathrm{m}$.

voltage of $2 \mathrm{~V}$ and a tip separation of $4 \mu \mathrm{m}$. This data is shown in Fig. 8 . The field gradient varies from a value of $\nabla E^{2}=10^{16} \mathrm{~V}^{2} \mathrm{~m}^{-3}$ in the centre (between the tips) to $\nabla E^{2}=10^{21} \mathrm{~V}^{2} \mathrm{~m}^{-3}$ at the tips. The initial movement of virus by DEP is seen to occur at a point approximately $\frac{1}{4}$ of the way across the gap, i.e. $1 \mu \mathrm{m}$ from the edges of the tips, and from the graph of Fig. 8 this point corresponds to an average value of $\nabla E^{2}=10^{17} \mathrm{~V}^{2} \mathrm{~m}^{-3}$.

The threshold field data can be analysed by plotting $\nabla E_{1}^{2}$ for each conductivity against the reciprocal of $\left.\operatorname{Re}\left\{\left(\varepsilon_{\mathrm{p}}^{*}-\varepsilon_{\mathrm{m}}^{*}\right) / \varepsilon_{\mathrm{m}}^{*}\right)\right\}$ and this is shown in Fig. 9. ${ }^{1}$ The crosses correspond to the experimental points and the line is a linear regression to the data. In constructing this plot the value of the field gradient at each value of threshold field was calculated from Fig. 8 at a point $1 \mu \mathrm{m}$ from the tips of the electrodes. The gradient of the plot is given by $\left(F_{1}\left(3 / 2 \pi a b c \varepsilon_{\mathrm{m}}\right)\right)$ where $F_{\mathrm{t}}$ is the threshold dielectrophoretic

${ }^{1}$ At $100 \mathrm{kHz}$ it is predicted from theory [20] that the TMV will always orient with the field for all conductivities. 


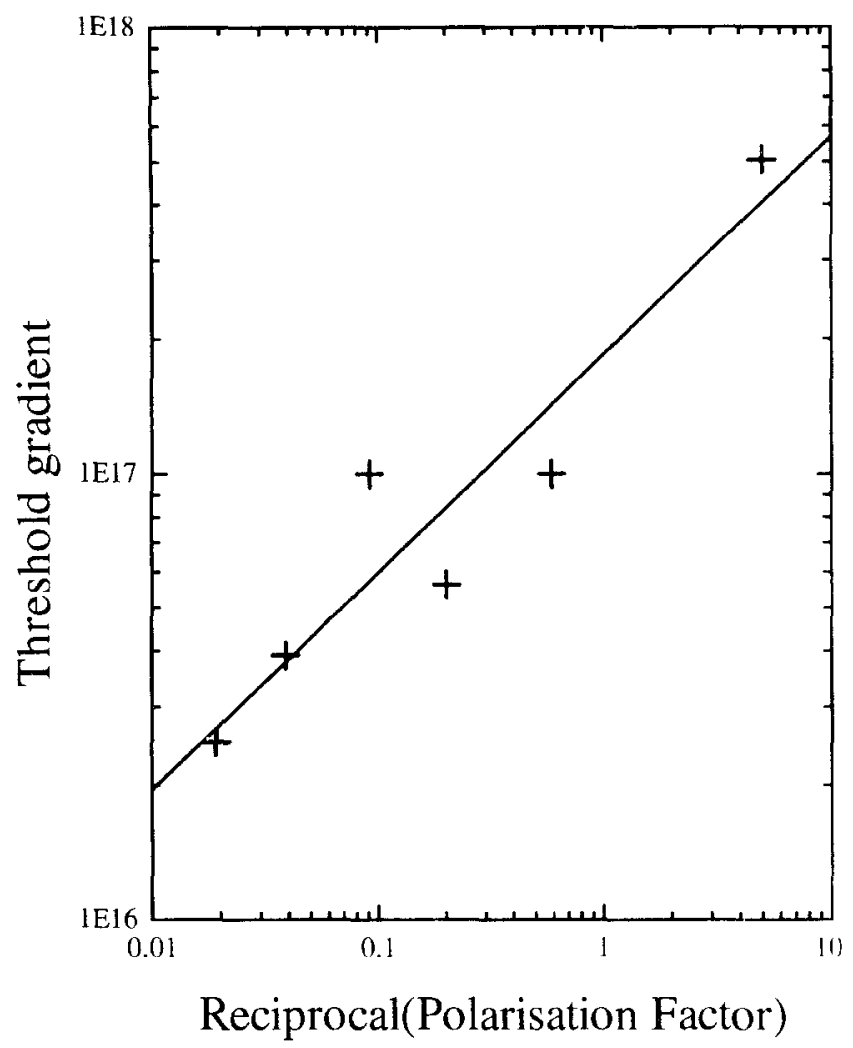

Fig. 9. The gradient of the threshold electric field strength required to initiate observable DFP plotted as a function of the reciprocal of the polarisation factor for the virus. Experimental points are indicalcd by crosses and the straight line is a least-squares fit to the datat.

force. The physical dimensions of the TMV particle are $9 \times 9 \times 140 \mathrm{~nm}$ and thus the minimum dielectrophoretic force which a single virion must experience in order 10 initiate movement can be estimated from the gradient to be $F_{\mathrm{t}}=5.8 \times 10^{-16} \mathrm{~N}$.

The high value of conductivity deduced for the TMV particle is likely to arise from the movement of counterions along the surface in addition to the movement of charge within the protein/RNA structure. These counterions may be locateci at the particle/medium interface (the so-called bound component of the double layer) or in the diffuse layer. If the volume of the TMV was adjusted to include the diffuse component of the double layer (equal to the Debye length). then at the lowest conductivity measured $\left(10^{-3} \mathrm{~S} \mathrm{~m}^{-1}\right)$ this would increase the volume of the particle by a fact.re of approximately 30 and the force by an equivalent amount. Even allowing for this; correction the estimated force is much lower than expected from simple diffusion arguments [1].

It is clear from Fig. 8 that the field gradient can greatly exceed the threshold value required to initiate DEP and also the value of the polarisability factor for TMV can 
reach 100 at low frequencies, indicating that positive dielectrophoretic collection of nano-scale particle should be possible over a wide range of conductivities, frequencies and particle size.

At higher frequencies $\left\{\left(\varepsilon_{\mathrm{p}}^{*}-\varepsilon_{\mathrm{m}}^{*}\right) / \varepsilon_{\mathrm{m}}^{*}\right\} \rightarrow\left\{\left(\varepsilon_{\mathrm{p}}-\varepsilon_{\mathrm{m}}\right) / \varepsilon_{\mathrm{m}}\right\}$ and negative DEP dominates. Whilst negative DEP was observed, the particles being distinctly repelled from the electrode tips, the value of $\operatorname{Re}\left\{\left(\varepsilon_{\mathrm{p}}^{*}-\varepsilon_{\mathrm{m}}^{*}\right) / \varepsilon_{\mathrm{m}}^{*}\right\} \approx-\frac{1}{4}$ is too small to give rise to a sufficiently large force to overcome Brownian motion and produce trapping of the particles in the low field regions of the electrode array (between the tips), since the low field region of the sawtooth electrodes have low values of electric field gradient. Polynomial electrodes have well-defined regions of low field gradients producing negative DEP funnels or traps [26,27], but we were unable to produce observable trapping of TMV under negative DEP in these electrode either, primarily owing to the low value of $\operatorname{Re}\left\{\left(\varepsilon_{\mathrm{p}}^{*}-\varepsilon_{\mathrm{m}}^{*}\right) / \varepsilon_{\mathrm{m}}^{*}\right\}$ at high frequencies.

\section{Conclusion}

This work has demonstrated that it is possible to manipulate TMV using nonuniform AC electric fields. The magnitude and direction of force on the particle has been derived and the experimental results are in accord with our theoretical predictions. The force is highly sensitive to frequency and medium conductivity and by implication a range of particles exhibiting different dielectric properties will experience different forces. At low frequencies the relative magnitude of the force on the TMV is dominated by the conductivity of the particle which in turn is dictated by the charge both in and on the surface of the virion. Thus, it is to be expected that a virion lacking in RNA might possess a different particle conductivity from normal TMV and thus experience a markedly different DEP force. Fractionation of nano-scale bioparticles according to the dielectrophoretic force may lead to potentially more sophisticated and sensitive separation technologies.

\section{Acknowledgements}

The authors wish to acknowledge EPSRC for a studentship for N.G. Green and BBSRC (Grant No. 17/T05313) for supporting this work. We acknowledge Dr. J.J. Milner for preparation of TMV, Dr. J.P.H. Burt (University of Wales, Bangor) for assistance with the electric field analysis and Prof. R. Pethig (University of Wales, Bangor) for valuable discussions.

\section{References}

[1] H.A. Pohl, The motion and precipitation of suspensoids in divergent electric fields, J. Appl. Phys. 22 (1951) 869-871.

[2] H.A. Pohl, Dielectrophoresis, Cambridge University Press, Cambridge, 1978. 
[3] N.G. Green, M.P. Hughes, W. Monaghan, H. Morgan, Large area multilayered electrode arrays for dielectrophoretic fractionation, Microelectronics 35 (1997) 421-424.

[4] T.B. Jones, Dielectrophoretic force calculations, J. Electrostatics 6 (1979) 69-82.

[5] T. Inoue, R. Pethig, T.A.K. Al-Ameen, J.P.H. Burt, J.A.R. Price, Dielectrophoretic behaviour of Micrococcus lysodeikticus and its protoplasts, J. Electrostatics 21 (1988) 215-223.

[6] R. Pethig, Application of a.c. electrical fields to the manipulation and characterisation of cells, in: I. Karube (Ed.), Automation in Biotechnology, Elsevier, Amsterdam, 1991, pp. 159-185.

[7] F.F. Becker, X.-B. Wang, Y. Huang, R. Pethig, J. Vykoukal, P.R.C. Gascoyne, Separation of human breast cancer cells from blood by differential dielectric affinity, Proc. Natl. Acad. Sci. USA 92 (1995) 860-864.

[8] H.A. Pohl, Non-uniform field effects in poorly conducting media, J. Electrochem. Soc. 107 (1960) 386-390.

[9] M. Washizu, S. Suzuki, O. Kurosawa, T. Nishizaka, T. Shinohara, Molecular dielectrophoresis of biopolymers, IEEE Trans. Ind. Appl. 30 (1994) 835-843.

[10] N.G. Green, H. Morgan, C.D.W. Wilkinson, J.J. Milner, Proc. St. Andrews Meeting, Animal and Cell Abstracts, St. Andrews, UK, Vol. C2.18, 1995, p. 77.

[11] G. Fuhr, ibid.

[12] T. Muller, A. Gerardino, Th. Schnelle, S.G. Shirley, F. Bordoni, G. De Gasperis, R. Leoni, G. Fuhr, Trapping of micrometer and submicrometer particles by high-frequency electric-fields and hydrodynamic-forces, J. Phys. D: Appl. Phys. 29 (1996) 340-349.

[13] T. Schnelle, T. Muller, S. Fiedler, S.G. Shirely, K. Ludwig, A. Hermann, G. Fuhr, Trapping of viruses in high-frequency electric field cages, Naturwissenschaften 83 (1996) 172-176.

[14] T. Muller, S. Fiedler, T. Schnelle, K. Ludwig, H. Jung, G. Fuhr, High frequency fields for trapping of viruses, Biotechnol. Techniques 10 (1996) 221-226.

[15] R. Clausius, Die mechanische Wärmetheorie, Vieweg, Braunschweig, Vol. II, 1879, p. 62ff.

[16] O.F. Mossotti, Discussione analitica, Mem. Soc. Ital. 14 (1850) 49.

[17] A.R. von Hippel, Dielectrics and Waves, Wiley, New York, 1954.

[18] J.A. Stratton, Electromagnetic Theory, McGraw-Hill, New York, 1941.

[19] T. Kakutani, S. Shibatani, M. Sugai, Electrorotation of non-spherical cells: theory for eilipsoidal cells with an arbitrary number of shells, Bioelectrochem. Bioenerg. 31 (1993) 131-145.

[20] R.D. Miller, T.B. Jones, Electro-orientation of ellipsoidal erythrocytes. Theory and experiment, Biophys. J. 64 (1993) 1588-1595.

[21] R.E.F. Mathews, Plant Virology, Academic Press, London, 1981.

[22] F. Van der Touw, J.W.H. Briede, M. Mandel, Electric permittivity of alfalfa mosaic virus in aqueous solutions, Biopolymers 12 (1973) 111-119.

[23] N.G. Green, H. Morgan, J.J. Milner, Manipulation and trapping of nanoscale bioparticles using dielectrophoresis, J. Biochem. Biophys. Methods, in press.

[24] M.A. Lauffer, Electro-optical effects in certain viruses, J. Am. Chem. Soc. 61 (1939) 2412-2416.

[25] C.T. O'Konski, B.H. Zimm, New methods for studying electrical orientation and relaxation effects in aqueous colloids: preliminary results with tobacco Mosaic Virus, Science 111 (1950) 113-116.

[26] Y. Huang, R. Pethig, Electrode design for negative dielectrophoresis, Meas. Sci. Technol. 2 (1991) $142-1146$.

[27] X.-B. Wang, Y. Huang, J.P.H. Burt, G.H. Markx, R. Pethig, Selective dielectrophoretic confinement of bioparticles in potential-energy wells, J. Phys. D: Appl. Phys. 26 (1993) 1278-1285. 